\title{
Complex Langevin dynamics at finite chemical potential: mean field analysis in the relativistic Bose gas
}

\author{
Gert Aarts \\ Department of Physics, Swansea University, Swansea, United Kingdom \\ Email: g.aarts@swan.ac.uk
}

\begin{abstract}
Stochastic quantization can potentially be used to simulate theories with a complex action due to a nonzero chemical potential. We study complex Langevin dynamics in the relativistic Bose gas analytically, using a mean field approximation. We concentrate on the region with a Silver Blaze problem and discuss convergence, stability, fixed points, and the severeness of the sign problem. The real distribution satisfying the extended Fokker-Planck equation is constructed and its nonlocal form is explained. Finally, we compare the mean field results in finite volume with the numerical data presented in Ref. [1].
\end{abstract}

KEYWORDS: Lattice Quantum Field Theory, Lattice QCD. 


\section{Contents}

1. Introduction 1

2. Relativistic Bose gas and Langevin dynamics 3

3. Ignoring interactions 5

3.1 Standard results 5

3.2 Continuous Langevin dynamics 7

3.3 Discretized Langevin dynamics 9

4. Fokker-Planck equation 10

5. Mean field approximation 12

6. Conclusion 16

A. Dispersion relation 17

\section{Introduction}

Theories with a complex action are not easy to solve numerically, since approaches based on importance sampling break down. This is commonly referred to as the sign problem. An important theory in this class is QCD at finite baryon chemical potential, with a complex fermion determinant satisfying $[\operatorname{det} M(\mu)]^{*}=\operatorname{det} M(-\mu){ }^{1}{ }^{1}$ Several methods have been devised to circumvent the sign problem in QCD, mostly at small chemical potential and in the vicinity of the crossover between the confined and the deconfined phase [2, 3, 4,5 , 6, 7, 8, 9, 10, 11, 12, 13, 14. For a detailed lattice QCD study of the sign problem at small chemical potential, see Ref. [15]. Considerable insight in the QCD sign problem has also been obtained with Random Matrix Theory [16, 17, 18, 19, 20, 21]. In some theories the sign problem can be eliminated altogether, using a reformulation in terms of different degrees of freedom [22, 23, 24].

Since stochastic quantization [25] does not rely on importance sampling, it can potentially be applied to theories with a complex action using complex Langevin dynamics [26, 27]. Studies in the 80's, however, have given mixed results, see e.g. Refs. [28, 29]. For an extensive review and more references, see Ref. [30]. Recently the approach was reconsidered as a method to solve nonequilibrium quantum fields dynamics in Minkowski spacetime 31, 32, 33]. It was shown that instabilities, which plagued earlier studies, can

\footnotetext{
${ }^{1}$ In case of a complex chemical potential, this relation becomes $[\operatorname{det} M(\mu)]^{*}=\operatorname{det} M\left(-\mu^{*}\right)$.
} 
be controlled by using small enough Langevin stepsizes. Moreover, insight in the convergence properties of the method can be obtained from features of classical flow diagrams. Other recent applications include PT symmetric theories [34] and unbounded actions [35]. In Ref. [36] we applied stochastic quantization to various theories with a nonzero chemical potential. In particular, we considered QCD with static quarks, in which the fermion determinant is approximated but the full gauge dynamics is preserved. First results on a $4^{4}$ lattice are encouraging. The required extension from $\mathrm{SU}(3)$ to $\mathrm{SL}(3, \mathbb{C})$ is discussed in detail.

The sign problem in QCD at finite chemical potential does not arise because of the anticommuting nature of the quark fields. Also in bosonic theories with a nonzero chemical potential and an action that behaves under complex conjugation as $S^{*}(\mu)=S(-\mu)$, the sign problem appears. In Ref. [1] we considered the relativistic Bose gas (a self interacting complex scalar field) in four dimensions in the presence of a chemical potential as one of the simplest examples of a relativistic field theory with a severe sign problem. Like QCD, this theory has a Silver Blaze problem [37]: at strictly zero temperature and small chemical potential, bulk physical observables are independent of the chemical potential, even though it enters explicitly in the microscopic dynamics. At larger chemical potential, the system enters a Bose condensed phase. The $\mu$ independence below onset and the formation of a state with nonzero density above onset is similar to what is expected to occur in QCD at zero temperature. It was demonstrated in Ref. [1] that complex Langevin dynamics reproduces the expected physics, on lattices of size $N^{4}$, with $N=4,6,8,10$. The sign problem was shown to be severe. However, no obstacles related to the sign problem, the Silver Blaze problem, or in taking the thermodynamic limit were encountered.

In this paper we complement the numerical study of Ref. [1] with a detailed analytical study in the mean field approximation. We concentrate on the region with the Silver Blaze problem. The paper is organized as follows. In Sec. 2 we remind the reader of the model and the corresponding complex Langevin equations. In order to prepare for the mean field analysis, we first discuss the case without interactions. In Sec. 3 we summarize the exact results in the free field limit, using standard field theory. Subsequently the free Langevin equations are solved analytically, both for continuous and discretized dynamics. We discuss convergence and stability properties. In Sec. 国 the stationary solution of the Fokker-Planck equation is given, again ignoring interactions, and shown to be in agreement with the solution of the Langevin equations in the limit of large Langevin time. ${ }^{2}$ The criterium for convergence is derived from this distribution as well. We find that the real probability distribution is highly nonlocal and explain why. In Sec. 5 interactions are included and the analysis is extended to the mean field approximation. We derive fixed points of the mean field Langevin equations at finite Langevin stepsize. Finally the mean field predictions in finite volume are compared with the nonperturbative results obtained by complex Langevin simulations [1]. The appendix contains a short remark about lattice dispersion relations.

\footnotetext{
${ }^{2}$ Recently, an interesting approach to study stationary solutions of complex Langevin dynamics was presented in Ref. 38].
} 


\section{Relativistic Bose gas and Langevin dynamics}

We consider a self-interacting complex scalar field in the presence of a chemical potential $\mu$, with the continuum action

$$
S=\int d^{4} x\left[\left|\partial_{\nu} \phi\right|^{2}+\left(m^{2}-\mu^{2}\right)|\phi|^{2}+\mu\left(\phi^{*} \partial_{4} \phi-\partial_{4} \phi^{*} \phi\right)+\lambda|\phi|^{4}\right] .
$$

The euclidean action is complex and satisfies $S^{*}(\mu)=S(-\mu)$. We take $m^{2}>0$, so that at vanishing and small $\mu$ the theory is in its symmetric phase.

We study this theory on the lattice, with the action

$$
S=\sum_{x}\left[\left(2 d+m^{2}\right) \phi_{x}^{*} \phi_{x}+\lambda\left(\phi_{x}^{*} \phi_{x}\right)^{2}-\sum_{\nu=1}^{4}\left(\phi_{x}^{*} e^{-\mu \delta_{\nu, 4}} \phi_{x+\hat{\nu}}+\phi_{x+\hat{\nu}}^{*} e^{\mu \delta_{\nu, 4}} \phi_{x}\right)\right] .
$$

As always, chemical potential is introduced as an imaginary constant vector potential in the temporal direction [39]. The number of euclidean dimensions is $d=4$, the lattice spacing $a_{\text {lat }} \equiv 1$, and the lattice four-volume is $\Omega=N_{s}^{3} N_{\tau}$, where $N_{s}\left(N_{\tau}\right)$ are the number of sites in a spatial (temporal) direction. We use periodic boundary conditions.

In order to formulate the complex Langevin equations for this theory, the complex field is first written in terms of two real fields $\phi_{a}(a=1,2)$ as $\phi=\frac{1}{\sqrt{2}}\left(\phi_{1}+i \phi_{2}\right)$. The lattice action then reads

$$
\begin{aligned}
S=\sum_{x}[ & \frac{1}{2} \\
& \left(2 d+m^{2}\right) \phi_{a, x}^{2}+\frac{\lambda}{4}\left(\phi_{a, x}^{2}\right)^{2}-\sum_{i=1}^{3} \phi_{a, x} \phi_{a, x+\hat{i}} \\
& \left.-\cosh \mu \phi_{a, x} \phi_{a, x+\hat{4}}+i \sinh \mu \varepsilon_{a b} \phi_{a, x} \phi_{b, x+\hat{4}}\right] .
\end{aligned}
$$

We use the antisymmetric tensor $\varepsilon_{a b}$, with $\varepsilon_{12}=-\varepsilon_{21}=1, \varepsilon_{11}=\varepsilon_{22}=0$, and summation over repeated indices is implied throughout.

Since the Boltzmann weight $e^{-S}$ in the partition function,

$$
Z=\int D \phi_{1} D \phi_{2} e^{-S}
$$

is complex, the theory has a sign problem and one cannot rely on importance sampling. Writing the weight as $\left|e^{-S}\right| e^{i \varphi}=e^{-S_{R}} e^{-i S_{I}}$, one may consider the phase quenched theory

$$
Z_{\mathrm{pq}}=\int D \phi_{1} D \phi_{2}\left|e^{-S}\right|=\int D \phi_{1} D \phi_{2} e^{-S_{R}}
$$

where $S_{R}$ is the real part of the action in Eq. (2.3), i.e. the term proportional to $\sinh \mu$ is dropped. By analysing the average phase factor in the phase quenched theory, $\left\langle e^{i \varphi}\right\rangle_{\mathrm{pq}}$, it was shown in Ref. [1] that this theory has a severe sign problem: at nonzero chemical potential the average phase factor goes to zero exponentially fast in the thermodynamic limit.

We use stochastic quantization. The Langevin equations for the fields $\phi_{a}$ read

$$
\frac{\partial}{\partial \theta} \phi_{a, x}(\theta)=-\frac{\delta S[\phi]}{\delta \phi_{a, x}(\theta)}+\eta_{a, x}(\theta)
$$


where $\theta$ is the Langevin time. The noise $\eta$ is Gaussian and normalized as

$$
\left\langle\eta_{a, x}(\theta)\right\rangle=0, \quad\left\langle\eta_{a, x}(\theta) \eta_{b, x^{\prime}}\left(\theta^{\prime}\right)\right\rangle=2 \delta_{a b} \delta_{x x^{\prime}} \delta\left(\theta-\theta^{\prime}\right) .
$$

Since the force in Eq. (2.6) is complex, the fields are complexified as

$$
\phi_{a} \rightarrow \phi_{a}^{\mathrm{R}}+i \phi_{a}^{\mathrm{I}} \quad(a=1,2) .
$$

The complex Langevin equations we consider in this paper then read

$$
\begin{aligned}
\frac{\partial}{\partial \theta} \phi_{a, x}^{\mathrm{R}}(\theta) & =K_{a, x}^{\mathrm{R}}(\theta)+\eta_{a, x}(\theta), \\
\frac{\partial}{\partial \theta} \phi_{a, x}^{\mathrm{I}}(\theta) & =K_{a, x}^{\mathrm{I}}(\theta) .
\end{aligned}
$$

The noise is chosen to be real. The drift terms are defined as

$$
\begin{aligned}
& K_{a, x}^{\mathrm{R}}=-\left.\operatorname{Re} \frac{\delta S}{\delta \phi_{a, x}}\right|_{\phi_{a} \rightarrow \phi_{a}^{\mathrm{R}}+i \phi_{a}^{\mathrm{I}}}, \\
& K_{a, x}^{\mathrm{I}}=-\left.\operatorname{Im} \frac{\delta S}{\delta \phi_{a, x}}\right|_{\phi_{a} \rightarrow \phi_{a}^{\mathrm{R}}+i \phi_{a}^{\mathrm{I}}},
\end{aligned}
$$

and read explicitly

$$
\begin{aligned}
K_{a, x}^{\mathrm{R}}= & -\left[2 d+m^{2}+\lambda\left(\phi_{b, x}^{\mathrm{R} 2}-\phi_{b, x}^{\mathrm{I} 2}\right)\right] \phi_{a, x}^{\mathrm{R}}+2 \lambda \phi_{b, x}^{\mathrm{R}} \phi_{b, x}^{\mathrm{I}} \phi_{a, x}^{\mathrm{I}}+\sum_{i}\left(\phi_{a, x+\hat{i}}^{\mathrm{R}}+\phi_{a, x-\hat{i}}^{\mathrm{R}}\right) \\
& +\cosh \mu\left(\phi_{a, x+\hat{4}}^{\mathrm{R}}+\phi_{a, x-\hat{4}}^{\mathrm{R}}\right)+\sinh \mu \varepsilon_{a b}\left(\phi_{b, x+\hat{4}}^{\mathrm{I}}-\phi_{b, x-\hat{4}}^{\mathrm{I}}\right), \\
K_{a, x}^{\mathrm{I}}= & -\left[2 d+m^{2}+\lambda\left(\phi_{b, x}^{\mathrm{R} 2}-\phi_{b, x}^{\mathrm{I} 2}\right)\right] \phi_{a, x}^{\mathrm{I}}-2 \lambda \phi_{b, x}^{\mathrm{R}} \phi_{b, x}^{\mathrm{I}} \phi_{a, x}^{\mathrm{R}}+\sum_{i}\left(\phi_{a, x+\hat{i}}^{\mathrm{I}}+\phi_{a, x-\hat{i}}^{\mathrm{I}}\right) \\
& +\cosh \mu\left(\phi_{a, x+\hat{4}}^{\mathrm{I}}+\phi_{a, x-\hat{4}}^{\mathrm{I}}\right)-\sinh \mu \varepsilon_{a b}\left(\phi_{b, x+\hat{4}}^{\mathrm{R}}-\phi_{b, x-\hat{4}}^{\mathrm{R}}\right) .
\end{aligned}
$$

Observables are written in terms of the complexified fields (2.8) as well. We consider the square of the field modulus,

$$
|\phi|^{2}=\frac{1}{2} \phi_{a}^{2} \rightarrow \frac{1}{2}\left(\phi_{a}^{\mathrm{R}^{2}}-\phi_{a}^{\mathrm{I}}\right)+i \phi_{a}^{\mathrm{R}} \phi_{a}^{\mathrm{I}}
$$

and the density $\langle n\rangle=(1 / \Omega) \partial \ln Z / \partial \mu$, given by $n=(1 / \Omega) \sum_{x} n_{x}$, with

$$
\begin{aligned}
n_{x} & =\left(\delta_{a b} \sinh \mu-i \varepsilon_{a b} \cosh \mu\right) \phi_{a, x} \phi_{b, x+\hat{4}} \\
& \rightarrow\left(\delta_{a b} \sinh \mu-i \varepsilon_{a b} \cosh \mu\right)\left(\phi_{a, x}^{\mathrm{R}} \phi_{b, x+\hat{4}}^{\mathrm{R}}-\phi_{a, x}^{\mathrm{I}} \phi_{b, x+\hat{4}}^{\mathrm{I}}+i\left[\phi_{a, x}^{\mathrm{R}} \phi_{b, x+\hat{4}}^{\mathrm{I}}+\phi_{a, x}^{\mathrm{I}} \phi_{b, x+\hat{4}}^{\mathrm{R}}\right]\right) .
\end{aligned}
$$

After complexification all observables have a real and imaginary part.

Employing that the noise is random, we observe that the Langevin equations have the following symmetry,

$$
\phi_{1, x}^{\mathrm{R}} \rightarrow-\phi_{1, x}^{\mathrm{R}}, \quad \phi_{2, x}^{\mathrm{R}} \rightarrow \phi_{2, x}^{\mathrm{R}}, \quad \phi_{1, x}^{\mathrm{I}} \rightarrow \phi_{1, x}^{\mathrm{I}}, \quad \phi_{2, x}^{\mathrm{I}} \rightarrow-\phi_{2, x}^{\mathrm{I}},
$$


for all $x$ and similar with 1 and 2 interchanged. Under this transformation, the drift terms change as

$$
K_{1, x}^{\mathrm{R}} \rightarrow-K_{1, x}^{\mathrm{R}}, \quad K_{2, x}^{\mathrm{R}} \rightarrow K_{2, x}^{\mathrm{R}}, \quad K_{1, x}^{\mathrm{I}} \rightarrow K_{1, x}^{\mathrm{I}}, \quad K_{2, x}^{\mathrm{I}} \rightarrow-K_{2, x}^{\mathrm{I}} .
$$

Correlation functions odd under this transformation should vanish after noise averaging, which implies that

$$
\left\langle\phi_{1, x}^{\mathrm{R}} \phi_{2, y}^{\mathrm{R}}\right\rangle=\left\langle\phi_{1, x}^{\mathrm{I}} \phi_{2, y}^{\mathrm{I}}\right\rangle=\left\langle\phi_{1, x}^{\mathrm{R}} \phi_{1, y}^{\mathrm{I}}\right\rangle=\left\langle\phi_{2, x}^{\mathrm{R}} \phi_{2, y}^{\mathrm{I}}\right\rangle=0 .
$$

The nonzero combinations are

$$
\left\langle\phi_{a, x}^{\mathrm{R}} \phi_{b, y}^{\mathrm{R}}\right\rangle \sim \delta_{a b}, \quad\left\langle\phi_{a, x}^{\mathrm{I}} \phi_{b, y}^{\mathrm{I}}\right\rangle \sim \delta_{a b}, \quad\left\langle\phi_{a, x}^{\mathrm{R}} \phi_{b, y}^{\mathrm{I}}\right\rangle \sim \varepsilon_{a b}
$$

Applying this to the expectation values of the observables in Eqs. 2.15, 2.16), we find that they are purely real. This is indeed what was observed numerically in Ref. [1].

\section{Ignoring interactions}

In order to set the stage for the mean field analysis, we first solve the Langevin dynamics without interactions $(\lambda=0)$, allowing for a detailed understanding of convergence and stability properties in the Silver Blaze regime.

\subsection{Standard results}

We start by summarizing the results obtained in the standard field theory approach (see e.g. Ref. [40]). After going to momentum space, according to

$$
\phi_{a, x}=\sum_{p} e^{i p x} \phi_{a, p}
$$

where $p_{i}=2 \pi n_{i} / N_{s}$, with $-N_{s} / 2<n_{i} \leq N_{s} / 2$, and $p_{4}=2 \pi n_{4} / N_{\tau}$, with $-N_{\tau} / 2<n_{4} \leq$ $N_{\tau} / 2$, the action (2.3) reads

$$
S=\sum_{p} \frac{1}{2} \phi_{a,-p}\left(\delta_{a b} A_{p}-\varepsilon_{a b} B_{p}\right) \phi_{b, p}=\sum_{p} \frac{1}{2} \phi_{a,-p} M_{a b, p} \phi_{b, p},
$$

where

$$
M_{p}=\left(\begin{array}{cc}
A_{p} & -B_{p} \\
B_{p} & A_{p}
\end{array}\right)
$$

and $^{3}$

$$
A_{p}=m^{2}+4 \sum_{i=1}^{3} \sin ^{2} \frac{p_{i}}{2}+2\left(1-\cosh \mu \cos p_{4}\right), \quad B_{p}=2 \sinh \mu \sin p_{4}
$$

\footnotetext{
${ }^{3}$ In the formal continuum limit $A_{p} \rightarrow m^{2}-\mu^{2}+p_{4}^{2}+\mathbf{p}^{2}, B_{p} \rightarrow 2 \mu p_{4}$.
} 
Note that $A_{-p}=A_{p}, B_{-p}=-B_{p}$, and that $M_{p}$ is nonhermitian. The phase quenched theory is obtained by taking $B_{p}=0$. Up to an irrelevant constant, the logarithm of the partition function is

$$
\ln Z=-\frac{1}{2} \sum_{p} \ln \operatorname{det} M_{p}=-\frac{1}{2} \sum_{p} \ln \left(A_{p}^{2}+B_{p}^{2}\right) .
$$

The observables we are interested in are given by

$$
\left\langle|\phi|^{2}\right\rangle=-\frac{1}{\Omega} \frac{\partial \ln Z}{\partial m^{2}}=\frac{1}{\Omega} \sum_{p} \frac{A_{p}}{A_{p}^{2}+B_{p}^{2}}
$$

and

$$
\langle n\rangle=\frac{1}{\Omega} \frac{\partial \ln Z}{\partial \mu}=-\frac{1}{\Omega} \sum_{p} \frac{A_{p} A_{p}^{\prime}+B_{p} B_{p}^{\prime}}{A_{p}^{2}+B_{p}^{2}},
$$

where $A^{\prime}=\partial A / \partial \mu=-2 \sinh \mu \cos p_{4}, B^{\prime}=\partial B / \partial \mu=2 \cosh \mu \sin p_{4}$.

As always, the severeness of the sign problem is estimated by the average phase factor in the phase quenched theory, given by the ratio of the partition functions of the full and phase quenched theories (2.4, 2.5),

$$
\left\langle e^{i \varphi}\right\rangle_{\mathrm{pq}}=\frac{Z}{Z_{\mathrm{pq}}}=e^{-\Omega \Delta f}
$$

where $\Delta f$, the difference between the corresponding free energy densities, is given by

$$
\Delta f=-\frac{1}{\Omega} \ln \frac{Z}{Z_{\mathrm{pq}}}=\frac{1}{2 \Omega} \sum_{p} \ln \frac{A_{p}^{2}+B_{p}^{2}}{A_{p}^{2}} .
$$

Note that this can be easily generalized to arbitrary powers of the phase factor in theories with nonvanishing phase factors. ${ }^{4}$

Finally, since the eigenvalues of $M_{p}$ in the action (3.2) are $A_{p} \pm i B_{p}$, the theory without interactions exists provided that $A_{p}>0$. This yields the standard stability criterium for a free Bose gas at finite chemical potential,

$$
A_{p}>0 \quad \Leftrightarrow \quad 4 \sinh ^{2} \frac{\mu}{2}<m^{2}
$$

corresponding to $\mu^{2}<m^{2}$ in the formal continuum limit. We restrict the analysis below therefore to the case that $A_{p}>0$; this is the Silver Blaze region.

\footnotetext{
${ }^{4}$ Define the partition function $Z_{\ell}=\int D \phi_{1} D \phi_{2}\left|e^{-S}\right| e^{i \ell \varphi}$. Then $\left\langle e^{i n \varphi}\right\rangle_{\ell}=Z_{n+\ell} / Z_{\ell}=\exp \left(-\Omega \Delta f_{n+\ell, \ell}\right)$, with

$$
\Delta f_{n+\ell, \ell}=-\frac{1}{\Omega} \ln \frac{Z_{n+\ell}}{Z_{\ell}}=\frac{1}{2 \Omega} \sum_{p} \ln \frac{A_{p}^{2}+(n+\ell)^{2} B_{p}^{2}}{A_{p}^{2}+\ell^{2} B_{p}^{2}} .
$$
}




\subsection{Continuous Langevin dynamics}

We now solve the complex Langevin equations to compare the outcome with the results given above. The Langevin equations $(2.9,2.10)$ read in momentum space

$$
\begin{aligned}
\frac{\partial}{\partial \theta} \phi_{a, p}^{\mathrm{R}}(\theta) & =K_{a, p}^{\mathrm{R}}(\theta)+\eta_{a, p}(\theta), \\
\frac{\partial}{\partial \theta} \phi_{a, p}^{\mathrm{I}}(\theta) & =K_{a, p}^{\mathrm{I}}(\theta),
\end{aligned}
$$

where the noise is normalized as

$$
\left\langle\eta_{a,-p}(\theta) \eta_{b, p^{\prime}}\left(\theta^{\prime}\right)\right\rangle=2 \delta_{a b} \delta_{p p^{\prime}} \delta\left(\theta-\theta^{\prime}\right)
$$

Ignoring interactions, we find for the drift terms

$$
\begin{aligned}
& K_{a, p}^{\mathrm{R}}=-A_{p} \phi_{a, p}^{\mathrm{R}}+i B_{p} \varepsilon_{a b} \phi_{b, p}^{\mathrm{I}}, \\
& K_{a, p}^{\mathrm{I}}=-A_{p} \phi_{a, p}^{\mathrm{I}}-i B_{p} \varepsilon_{a b} \phi_{b, p}^{\mathrm{R}},
\end{aligned}
$$

where $A_{p}$ and $B_{p}$ are defined in Eq. (3.4) above. In terms of

$$
\mathcal{M}_{p}=\left(\begin{array}{cccc}
A_{p} & 0 & 0 & -i B_{p} \\
0 & A_{p} & i B_{p} & 0 \\
0 & i B_{p} & A_{p} & 0 \\
-i B_{p} & 0 & 0 & A_{p}
\end{array}\right), \quad \Phi_{p}=\left(\begin{array}{c}
\phi_{1, p}^{\mathrm{R}} \\
\phi_{1, p}^{\mathrm{I}} \\
\phi_{2, p}^{\mathrm{R}} \\
\phi_{2, p}^{\mathrm{I}}
\end{array}\right), \quad \Xi_{p}=\left(\begin{array}{c}
\eta_{1, p} \\
0 \\
\eta_{2, p} \\
0
\end{array}\right)
$$

the Langevin dynamics is written as

$$
\frac{\partial}{\partial \theta} \Phi_{p}=-\mathcal{M}_{p} \Phi_{p}+\Xi_{p}
$$

The matrix $\mathcal{M}$ can be diagonalized by an orthogonal transformation and has doubly degenerate eigenvalues $\lambda_{p}=A_{p} \pm i B_{p}$. The solution of the Langevin equations is

$$
\begin{aligned}
\phi_{a, p}^{\mathrm{R}}(\theta)= & e^{-A_{p} \theta}\left[\cos \left(B_{p} \theta\right) \phi_{a, p}^{\mathrm{R}}(0)+i \sin \left(B_{p} \theta\right) \varepsilon_{a b} \phi_{b, p}^{\mathrm{I}}(0)\right] \\
& +\int_{0}^{\theta} d s e^{-A_{p}(\theta-s)} \cos \left[B_{p}(\theta-s)\right] \eta_{a, p}(s), \\
\phi_{a, p}^{\mathrm{I}}(\theta)= & e^{-A_{p} \theta}\left[\cos \left(B_{p} \theta\right) \phi_{a, p}^{\mathrm{I}}(0)-i \sin \left(B_{p} \theta\right) \varepsilon_{a b} \phi_{b, p}^{\mathrm{R}}(0)\right] \\
& -i \int_{0}^{\theta} d s e^{-A_{p}(\theta-s)} \sin \left[B_{p}(\theta-s)\right] \varepsilon_{a b} \eta_{b, p}(s),
\end{aligned}
$$

where $\phi_{a, p}^{\mathrm{R}, \mathrm{I}}(0)$ denote the initial conditions.

We are now in a position to discuss the convergence properties of the Langevin process in the limit of large Langevin time. First we note that there is independence of initial conditions provided that $A_{p}>0$, i.e. in the region of interest here. Taking $\phi_{a, p}^{\mathrm{R}, \mathrm{I}}(0)=0$, we find for the two-point functions, after using Eq. (3.13) and performing the Langevin time 
integrals,

$$
\begin{aligned}
\left\langle\phi_{a,-p}^{\mathrm{R}}(\theta) \phi_{b, p^{\prime}}^{\mathrm{R}}(\theta)\right\rangle= & \frac{1}{2 A_{p}} \frac{\delta_{a b} \delta_{p p^{\prime}}}{A_{p}^{2}+B_{p}^{2}}\left(2 A_{p}^{2}+B_{p}^{2}\right. \\
& -e^{-2 A_{p} \theta}\left[A_{p}^{2}+B_{p}^{2}+A_{p}^{2} \cos \left(2 B_{p} \theta\right)-A_{p} B_{p} \sin \left(2 B_{p} \theta\right)\right] \\
\left\langle\phi_{a,-p}^{\mathrm{I}}(\theta) \phi_{b, p^{\prime}}^{\mathrm{I}}(\theta)\right\rangle= & \frac{1}{2 A_{p}} \frac{\delta_{a b} \delta_{p p^{\prime}}}{A_{p}^{2}+B_{p}^{2}}\left(B^{2}\right. \\
& \left.-e^{-2 A_{p} \theta}\left[A_{p}^{2}+B_{p}^{2}-A_{p}^{2} \cos \left(2 B_{p} \theta\right)+A_{p} B_{p} \sin \left(2 B_{p} \theta\right)\right]\right) \\
\left\langle\phi_{a,-p}^{\mathrm{R}}(\theta) \phi_{b, p^{\prime}}^{\mathrm{I}}(\theta)\right\rangle= & \frac{i}{2} \frac{\varepsilon_{a b} \delta_{p p^{\prime}}}{A_{p}^{2}+B_{p}^{2}}\left(B_{p}-e^{-2 A_{p} \theta}\left[B_{p} \cos \left(2 B_{p} \theta\right)+A_{p} \sin \left(2 B_{p} \theta\right)\right]\right)
\end{aligned}
$$

Most of the terms vanish in the limit that $\theta \rightarrow \infty$, again provided that $A_{p}>0$. The surviving terms are

$$
\begin{aligned}
& \lim _{\theta \rightarrow \infty}\left\langle\phi_{a,-p}^{\mathrm{R}}(\theta) \phi_{b, p^{\prime}}^{\mathrm{R}}(\theta)\right\rangle \equiv\left\langle\phi_{a,-p}^{\mathrm{R}} \phi_{b, p^{\prime}}^{\mathrm{R}}\right\rangle=\delta_{a b} \delta_{p p^{\prime}} \frac{1}{2 A_{p}} \frac{2 A_{p}^{2}+B_{p}^{2}}{A_{p}^{2}+B_{p}^{2}}, \\
& \lim _{\theta \rightarrow \infty}\left\langle\phi_{a,-p}^{\mathrm{I}}(\theta) \phi_{b, p^{\prime}}^{\mathrm{I}}(\theta)\right\rangle \equiv\left\langle\phi_{a,-p}^{\mathrm{I}} \phi_{b, p^{\prime}}^{\mathrm{I}}\right\rangle=\delta_{a b} \delta_{p p^{\prime}} \frac{1}{2 A_{p}} \frac{B_{p}^{2}}{A_{p}^{2}+B_{p}^{2}}, \\
& \lim _{\theta \rightarrow \infty}\left\langle\phi_{a,-p}^{\mathrm{R}}(\theta) \phi_{b, p^{\prime}}^{\mathrm{I}}(\theta)\right\rangle \equiv\left\langle\phi_{a,-p}^{\mathrm{R}} \phi_{b, p^{\prime}}^{\mathrm{I}}\right\rangle=\varepsilon_{a b} \delta_{p p^{\prime}} \frac{i}{2} \frac{B_{p}}{A_{p}^{2}+B_{p}^{2}} .
\end{aligned}
$$

The structure of these two-point functions is in agreement with the symmetry (2.17, 2.20) discussed above.

For the observables we find the following. The square of the field modulus (2.15) is given by

$$
\begin{aligned}
\left\langle|\phi|^{2}\right\rangle & =\frac{1}{2 \Omega} \sum_{p}\left\langle\phi_{a,-p}^{\mathrm{R}} \phi_{a, p}^{\mathrm{R}}-\phi_{a,-p}^{\mathrm{I}} \phi_{a, p}^{\mathrm{I}}+2 i \phi_{a,-p}^{\mathrm{R}} \phi_{a, p}^{\mathrm{I}}\right\rangle \\
& =\frac{1}{\Omega} \sum_{p} \frac{A_{p}}{A_{p}^{2}+B_{p}^{2}},
\end{aligned}
$$

which agrees with Eq. (3.6). After going to momentum space, the density (2.16) reads

$$
\begin{aligned}
\langle n\rangle & =\frac{1}{\Omega} \sum_{p}\left(\delta_{a b} \sinh \mu \cos p_{4}+\varepsilon_{a b} \cosh \mu \sin p_{4}\right)\left\langle\phi_{a,-p}^{\mathrm{R}} \phi_{b, p}^{\mathrm{R}}-\phi_{a,-p}^{\mathrm{I}} \phi_{b, p}^{\mathrm{I}}+2 i \phi_{a,-p}^{\mathrm{R}} \phi_{b, p}^{\mathrm{I}}\right\rangle \\
& =\frac{2}{\Omega} \sum_{p}\left[\sinh \mu \cos p_{4} \frac{A_{p}}{A_{p}^{2}+B_{p}^{2}}-\cosh \mu \sin p_{4} \frac{B_{p}}{A_{p}^{2}+B_{p}^{2}}\right]
\end{aligned}
$$

which agrees with Eq. (3.7). Note that all two-point functions in Eq. (3.21) contribute to this answer.

We conclude therefore that the Langevin process is independent of initial conditions and converges to the correct result in the limit of infinite Langevin time, provided that $A_{p}>0$, as required in the Silver Blaze region. Moreover, the complexification is essential, as exemplified by the observables above. 


\subsection{Discretized Langevin dynamics}

We proceed by briefly considering the Langevin process after discretizing Langevin time as $\theta=n \epsilon$, where $\epsilon$ is the Langevin time step. The discretized Langevin equations are

$$
\begin{aligned}
& \phi_{a, p}^{\mathrm{R}}(n+1)=\phi_{a, p}^{\mathrm{R}}(n)+\epsilon K_{a, p}^{\mathrm{R}}(n)+\sqrt{\epsilon} \eta_{a, p}(n), \\
& \phi_{a, p}^{\mathrm{I}}(n+1)=\phi_{a, p}^{\mathrm{I}}(n)+\epsilon K_{a, p}^{\mathrm{I}}(n),
\end{aligned}
$$

and the noise obeys $\left\langle\eta_{a,-p}(n) \eta_{b, p^{\prime}}\left(n^{\prime}\right)\right\rangle=2 \delta_{n n^{\prime}} \delta_{a b} \delta_{p p^{\prime}}$. In the notation of Eq. (3.16), these equations are summarized as

$$
\Phi_{p}(n+1)=\left(1-\epsilon \mathcal{M}_{p}\right) \Phi_{p}(n)+\sqrt{\epsilon} \Xi_{p}(n)
$$

and solved by

$$
\Phi_{p}(n)=\left(1-\epsilon \mathcal{M}_{p}\right)^{n} \Phi_{p}(0)+\sqrt{\epsilon} \sum_{i=0}^{n-1}\left(1-\epsilon \mathcal{M}_{p}\right)^{n-1-i} \Xi_{p}(i),
$$

where $\Phi_{p}(0)$ is the initial condition. Convergence is determined by the eigenvalues of $\mathcal{M}_{p}$. This yields the condition

$$
\left|1-\epsilon \lambda_{p}\right|<1, \quad \lambda_{p}=A_{p} \pm i B_{p}
$$

resulting in the constraint

$$
A_{p}-\frac{\epsilon}{2}\left(A_{p}^{2}+B_{p}^{2}\right)>0 .
$$

We find that the convergence criterium is modified by an explicit stepsize dependence. However, this restriction is not special for the complex Langevin process [41, 30]. Consider real Langevin dynamics at zero $\mu$ or in the phase quenched theory. In both cases $B_{p}=0$ and the criterium reads

$$
0<\frac{\epsilon}{2} A_{p}<1
$$

Since $A_{p}$ is maximal at the edge of the Brillouin zone $(p=\pi)$, this yields the modest constraint (for $\mu=0$ )

$$
\epsilon<\frac{2}{4 d+m^{2}}
$$

At nonzero chemical potential, this constraint is modified to

$$
\epsilon<\frac{2}{4 d+m^{2}+2(\cosh \mu-1)}
$$

both in the full and the phase quenched theory. In the Silver Blaze region, where $\mu$ is bounded, this leads to only a slightly stronger bound on $\epsilon$. However, since this bound is determined by ultraviolet modes at the scale of the lattice cutoff, it is likely that a similar constraint holds in the high-density phase as well. In the limit that $\mu \gg 1$, exponentially small stepsizes would eventually be required. It should be noted, however, that for such large chemical potentials lattice artefacts are severe.

The solution (3.27) can be used to study finite stepsize effects in two-point functions at infinite Langevin time. We come back to this below using a more elegant approach based on fixed points of the Langevin equations. 


\section{Fokker-Planck equation}

In order to better understand the Langevin process, we now study properties of the associated distributions.

Consider first the Langevin process (2.6) and the distribution $P[\phi, \theta]$, defined via

$$
\langle O[\phi, \theta]\rangle_{\eta}=\int D \phi P[\phi, \theta] O[\phi],
$$

where the brackets on the LHS denote noise averaging. The distribution satisfies the Fokker-Planck equation (in continuous Langevin time)

$$
\frac{\partial P[\phi, \theta]}{\partial \theta}=\sum_{x} \frac{\delta}{\delta \phi_{a, x}(\theta)}\left(\frac{\delta}{\delta \phi_{a, x}(\theta)}+\frac{\delta S[\phi]}{\delta \phi_{a, x}(\theta)}\right) P[\phi, \theta] .
$$

As always, the index $a=1,2$ is summed over. The stationary solution,

$$
P[\phi] \sim e^{-S[\phi]},
$$

always exists. However, since the action is complex, this is not the probability distribution for the complex Langevin process.

More relevant for the complexified process (2.9, 2.10) we consider here, is the real distribution $\rho\left[\phi^{\mathrm{R}}, \phi^{\mathrm{I}}, \theta\right]$, defined via [26]

$$
\left\langle O\left[\phi^{\mathrm{R}}+i \phi^{\mathrm{I}}, \theta\right]\right\rangle_{\eta}=\int D \phi^{\mathrm{R}} D \phi^{\mathrm{I}} \rho\left[\phi^{\mathrm{R}}, \phi^{\mathrm{I}}, \theta\right] O\left[\phi^{\mathrm{R}}+i \phi^{\mathrm{I}}\right] .
$$

This distribution satisfies the extended Fokker-Planck equation

$$
\frac{\partial \rho\left[\phi^{\mathrm{R}}, \phi^{\mathrm{I}}, \theta\right]}{\partial \theta}=\sum_{x}\left[\frac{\delta}{\delta \phi_{a, x}^{\mathrm{R}}(\theta)}\left(\frac{\delta}{\delta \phi_{a, x}^{\mathrm{R}}(\theta)}-K_{a, x}^{\mathrm{R}}(\theta)\right)-\frac{\delta}{\delta \phi_{a, x}^{\mathrm{I}}(\theta)} K_{a, x}^{\mathrm{I}}(\theta)\right] \rho\left[\phi^{\mathrm{R}}, \phi^{\mathrm{I}}, \theta\right] .
$$

If stochastic quantization is applicable for complex actions, the two expectation values (4.1) and (4.4) should be equal [26].

We focus on the stationary solution of Eq. (4.5) and henceforth drop the $\theta$ dependence. Ignoring again interactions, the stationary solution should satisfy

$$
\sum_{p}\left[\frac{\delta}{\delta \phi_{a, p}^{\mathrm{R}}}\left(\frac{\delta}{\delta \phi_{a,-p}^{\mathrm{R}}}-K_{a, p}^{\mathrm{R}}\right)-\frac{\delta}{\delta \phi_{a, p}^{\mathrm{I}}} K_{a, p}^{\mathrm{I}}\right] \rho\left[\phi^{\mathrm{R}}, \phi^{\mathrm{I}}\right]=0,
$$

where the drift terms $K_{a, p}^{\mathrm{R}, \mathrm{I}}$ were given in Eqs. (3.14, 3.15). Explicitly, this reads

$$
\begin{aligned}
\sum_{p}[ & \frac{\delta}{\delta \phi_{a, p}^{\mathrm{R}}} \frac{\delta}{\delta \phi_{a,-p}^{\mathrm{R}}}+\left(A_{p} \phi_{a, p}^{\mathrm{R}}-i B_{p} \varepsilon_{a b} \phi_{b, p}^{\mathrm{I}}\right) \frac{\delta}{\delta \phi_{a, p}^{\mathrm{R}}} \\
& \left.+\left(A_{p} \phi_{a, p}^{\mathrm{I}}+i B_{p} \varepsilon_{a b} \phi_{b, p}^{\mathrm{R}}\right) \frac{\delta}{\delta \phi_{a, p}^{\mathrm{I}}}+2 A_{p}\right] \rho\left[\phi^{\mathrm{R}}, \phi^{\mathrm{I}}\right]=0 .
\end{aligned}
$$


Based on the structure of the equation, the solution can be written as

$$
\rho\left[\phi^{\mathrm{R}}, \phi^{\mathrm{I}}\right]=N \exp \left[-\sum_{p}\left(\alpha_{p} \phi_{a,-p}^{\mathrm{R}} \phi_{a, p}^{\mathrm{R}}+\beta_{p} \phi_{a,-p}^{\mathrm{I}} \phi_{a, p}^{\mathrm{I}}+2 i \varepsilon_{a b} \gamma_{p} \phi_{a,-p}^{\mathrm{R}} \phi_{b, p}^{\mathrm{I}}\right)\right],
$$

where $N$ is a normalization constant. Inserting this expression in Eq. (4.7) yields the coefficients

$$
\alpha_{p}=A_{p}, \quad \beta_{p}=\frac{A_{p}}{B_{p}^{2}}\left(2 A_{p}^{2}+B_{p}^{2}\right), \quad \gamma_{p}=\frac{A_{p}^{2}}{B_{p}} .
$$

We have therefore found the stationary distribution corresponding to the complex Langevin process in the noninteracting case. ${ }^{5}$ Note that since $\gamma_{-p}=-\gamma_{p}$, the distribution is real in real space, as it should be.

We now verify that this stationary solution is indeed the distribution corresponding to the Langevin process in the limit of infinite Langevin time. Performing the Gaussian integrals, we find the partition function

$$
Z=\prod_{p} \int d \phi_{p}^{\mathrm{R}} d \phi_{p}^{\mathrm{I}} \rho\left[\phi^{\mathrm{R}}, \phi^{\mathrm{I}}\right]=\mathcal{N} \prod_{p} \frac{1}{\alpha_{p} \beta_{p}-\gamma_{p}^{2}},
$$

where $\mathcal{N}$ is an irrelevant constant and

$$
\alpha_{p} \beta_{p}-\gamma_{p}^{2}=\frac{A_{p}^{2}}{B_{p}^{2}}\left(A_{p}^{2}+B_{p}^{2}\right)>0 .
$$

The two-point functions that follow from this distribution are

$$
\begin{aligned}
\left\langle\phi_{a,-p}^{\mathrm{R}} \phi_{a, p}^{\mathrm{R}}\right\rangle & =-\frac{\partial \ln Z}{\partial \alpha_{p}}=\frac{\beta_{p}}{\alpha_{p} \beta_{p}-\gamma_{p}^{2}}=\frac{1}{A_{p}} \frac{2 A_{p}^{2}+B_{p}^{2}}{A_{p}^{2}+B_{p}^{2}}, \\
\left\langle\phi_{a,-p}^{\mathrm{I}} \phi_{a, p}^{\mathrm{I}}\right\rangle & =-\frac{\partial \ln Z}{\partial \beta_{p}}=\frac{\alpha_{p}}{\alpha_{p} \beta_{p}-\gamma_{p}^{2}}=\frac{1}{A_{p}} \frac{B_{p}^{2}}{A_{p}^{2}+B_{p}^{2}}, \\
2 i \varepsilon_{a b}\left\langle\phi_{a,-p}^{\mathrm{R}} \phi_{b, p}^{\mathrm{I}}\right\rangle & =-\frac{\partial \ln Z}{\partial \gamma_{p}}=\frac{-2 \gamma_{p}}{\alpha_{p} \beta_{p}-\gamma_{p}^{2}}=\frac{-2 B_{p}}{A_{p}^{2}+B_{p}^{2}} .
\end{aligned}
$$

These agree exactly with the results obtained by solving the Langevin equation, cf. Eq. (3.21).

The theory with the probability distribution $\rho\left[\phi^{\mathrm{R}}, \phi^{\mathrm{I}}\right]$ exists provided that the eigenvalues of the quadratic form in Eq. (4.8) are positive. We find the eigenvalues to be

$$
\begin{aligned}
\lambda_{p} & =\frac{1}{2}\left(\alpha_{p}+\beta_{p} \pm \sqrt{\left(\alpha_{p}-\beta_{p}\right)^{2}+4 \gamma_{p}^{2}}\right) \\
& =\frac{A_{p}}{B_{p}^{2}} \sqrt{A_{p}^{2}+B_{p}^{2}}\left(\sqrt{A_{p}^{2}+B_{p}^{2}} \pm A_{p}\right) .
\end{aligned}
$$

These are positive provided that $A_{p}>0$. The criterium that determines the convergence of the Langevin dynamics also emerges in the stationary solution of the extended FokkerPlanck equation, as expected.

\footnotetext{
${ }^{5}$ See Refs. [12, 13] for other examples.
} 
Let us discuss some more properties of the distribution (4.8). First we note that the distribution is highly nonlocal in real space and does not allow for e.g. a derivative expansion, due to the division by $B_{p}=2 \sinh \mu \sin p_{4}$ in the coefficients (4.9). We find therefore that the complexity of the original local weight $e^{-S}$ has been traded for the nonlocality of the real probability distribution. However, this nonlocal behaviour is expected: it follows from the Langevin equations that the modes with $p_{4}=0$ are purely real, i.e. $\phi_{a,\left(p_{4}=0, \mathbf{p}\right)}^{\mathrm{I}}=0$. This is enforced in the probability distribution by the singular behaviour as $p_{4} \rightarrow 0$. For the same reason the limit $\mu \rightarrow 0$ is singular, since there is no need to complexify the dynamics in this case and the distribution for the $\phi^{\mathrm{I}}$ modes should reduce to a delta function, $\delta\left(\phi^{\mathrm{I}}\right)$. These considerations fix the dependence on $B_{p}$.

In conclusion, we have found the stationary solution of the extended Fokker-Planck distribution. The real distribution is nonlocal and singular in the limit that $\mu, p_{4} \rightarrow 0$.

\section{Mean field approximation}

We now return to the interacting theory, with discretized Langevin time $\theta=n \epsilon$, and consider the two-point functions

$$
\begin{aligned}
& G_{a b, p}^{\mathrm{RR}}(n)=\left\langle\phi_{a,-p}^{\mathrm{R}}(n) \phi_{b, p}^{\mathrm{R}}(n)\right\rangle, \\
& G_{a b, p}^{\mathrm{II}}(n)=\left\langle\phi_{a,-p}^{\mathrm{I}}(n) \phi_{b, p}^{\mathrm{I}}(n)\right\rangle, \\
& G_{a b, p}^{\mathrm{RI}}(n)=\left\langle\phi_{a,-p}^{\mathrm{R}}(n) \phi_{b, p}^{\mathrm{I}}(n)\right\rangle .
\end{aligned}
$$

Using the Langevin equations (3.24, 3.25), we find that these correlation functions evolve according to

$$
\begin{aligned}
G_{a b, p}^{\mathrm{RR}}(n+1)= & G_{a b, p}^{\mathrm{RR}}(n)+\epsilon\left\langle\phi_{a,-p}^{\mathrm{R}}(n) K_{b, p}^{\mathrm{R}}(n)+K_{a,-p}^{\mathrm{R}}(n) \phi_{b, p}^{\mathrm{R}}(n)\right\rangle \\
& +\epsilon^{2}\left\langle K_{a,-p}^{\mathrm{R}}(n) K_{b, p}^{\mathrm{R}}(n)\right\rangle+\epsilon\left\langle\eta_{a,-p} \eta_{b, p}\right\rangle, \\
G_{a b, p}^{\mathrm{II}}(n+1)= & G_{a b, p}^{\mathrm{II}}(n)+\epsilon\left\langle\phi_{a,-p}^{\mathrm{I}}(n) K_{b, p}^{\mathrm{I}}(n)+K_{a,-p}^{\mathrm{I}}(n) \phi_{b, p}^{\mathrm{I}}(n)\right\rangle \\
& +\epsilon^{2}\left\langle K_{a,-p}^{\mathrm{I}}(n) K_{b, p}^{\mathrm{I}}(n)\right\rangle, \\
G_{a b, p}^{\mathrm{RI}}(n+1)= & G_{a b, p}^{\mathrm{RI}}(n)+\epsilon\left\langle\phi_{a,-p}^{\mathrm{R}}(n) K_{b, p}^{\mathrm{I}}(n)+K_{a,-p}^{\mathrm{R}}(n) \phi_{b, p}^{\mathrm{I}}(n)\right\rangle \\
& +\epsilon^{2}\left\langle K_{a,-p}^{\mathrm{R}}(n) K_{b, p}^{\mathrm{I}}(n)\right\rangle .
\end{aligned}
$$

Here we used that $\left\langle\eta_{a,-p}(n) \phi_{b, p}^{\mathrm{R}, \mathrm{I}}(n)\right\rangle=0$, since the fields at time $n$ do not depend on the noise at time $n$. The terms proportional to $\epsilon^{2}$ are finite stepsize corrections. We then look for fixed points of the Langevin equations, and put

$$
G_{a b, p}^{\mathrm{RR}}(n+1)=G_{a b, p}^{\mathrm{RR}}(n),
$$

etc. This yields the fixed point equations

$$
\begin{aligned}
& \left\langle\phi_{a,-p}^{\mathrm{R}} K_{b, p}^{\mathrm{R}}+K_{a,-p}^{\mathrm{R}} \phi_{b, p}^{\mathrm{R}}\right\rangle+\epsilon\left\langle K_{a,-p}^{\mathrm{R}} K_{b, p}^{\mathrm{R}}\right\rangle=-2 \delta_{a b}, \\
& \left\langle\phi_{a,-p}^{\mathrm{I}} K_{b, p}^{\mathrm{I}}+K_{a,-p}^{\mathrm{I}} \phi_{b, p}^{\mathrm{I}}\right\rangle+\epsilon\left\langle K_{a,-p}^{\mathrm{I}} K_{b, p}^{\mathrm{I}}\right\rangle=0, \\
& \left\langle\phi_{a,-p}^{\mathrm{R}} K_{b, p}^{\mathrm{I}}+K_{a,-p}^{\mathrm{R}} \phi_{b, p}^{\mathrm{I}}\right\rangle+\epsilon\left\langle K_{a,-p}^{\mathrm{R}} K_{b, p}^{\mathrm{I}}\right\rangle=0 .
\end{aligned}
$$


To implement a mean field approximation and find a self-consistent set for the twopoint functions (5.1), we factorize the interaction terms. Consider for example the term $\phi_{b, x}^{\mathrm{R}} \phi_{b, x}^{\mathrm{I}} \phi_{a, x}^{\mathrm{R}}$ appearing in the drift term (2.14). We write

$$
\phi_{b, x}^{\mathrm{R}} \phi_{b, x}^{\mathrm{I}} \phi_{a, x}^{\mathrm{R}} \rightarrow\left\langle\phi_{b, x}^{\mathrm{R}} \phi_{b, x}^{\mathrm{I}}\right\rangle \phi_{a, x}^{\mathrm{R}}+\left\langle\phi_{b, x}^{\mathrm{R}} \phi_{a, x}^{\mathrm{R}}\right\rangle \phi_{b, x}^{\mathrm{I}}+\left\langle\phi_{b, x}^{\mathrm{I}} \phi_{a, x}^{\mathrm{R}}\right\rangle \phi_{b, x}^{\mathrm{R}} .
$$

Using the notation

$$
\begin{aligned}
G_{a b}^{\mathrm{RR}}(n) & =\left\langle\phi_{a, x}^{\mathrm{R}}(n) \phi_{b, x}^{\mathrm{R}}(n)\right\rangle=\frac{1}{\Omega} \sum_{p} G_{a b, p}^{\mathrm{RR}}(n), \\
G_{a b}^{\mathrm{II}}(n) & =\left\langle\phi_{a, x}^{\mathrm{I}}(n) \phi_{b, x}^{\mathrm{I}}(n)\right\rangle=\frac{1}{\Omega} \sum_{p} G_{a b, p}^{\mathrm{II}}(n), \\
G_{a b}^{\mathrm{RI}}(n) & =\left\langle\phi_{a, x}^{\mathrm{R}}(n) \phi_{b, x}^{\mathrm{I}}(n)\right\rangle=\frac{1}{\Omega} \sum_{p} G_{a b, p}^{\mathrm{RI}}(n),
\end{aligned}
$$

the drift terms in the mean field approximation read

$$
\begin{aligned}
K_{a, p}^{\mathrm{R}}= & -\left[A_{p}+\lambda\left(G_{b b}^{\mathrm{RR}}-G_{b b}^{\mathrm{II}}\right)\right] \phi_{a, p}^{\mathrm{R}}-2 \lambda\left(G_{a b}^{\mathrm{RR}}-G_{a b}^{\mathrm{II}}\right) \phi_{b, p}^{\mathrm{R}} \\
& +\left[i B_{p} \varepsilon_{a b}+2 \lambda\left(G_{a b}^{\mathrm{RI}}+G_{b a}^{\mathrm{RI}}\right)\right] \phi_{b, p}^{\mathrm{I}}+2 \lambda G_{b b}^{\mathrm{RI}} \phi_{a, p}^{\mathrm{I}}, \\
K_{a, p}^{\mathrm{I}}= & -\left[A_{p}+\lambda\left(G_{b b}^{\mathrm{RR}}-G_{b b}^{\mathrm{II}}\right)\right] \phi_{a, p}^{\mathrm{I}}-2 \lambda\left(G_{a b}^{\mathrm{RR}}-G_{a b}^{\mathrm{I}}\right) \phi_{b, p}^{\mathrm{I}} \\
& -\left[i B_{p} \varepsilon_{a b}+2 \lambda\left(G_{a b}^{\mathrm{RI}}+G_{b a}^{\mathrm{RI}}\right)\right] \phi_{b, p}^{\mathrm{R}}-2 \lambda G_{b b}^{\mathrm{RI}} \phi_{a, p}^{\mathrm{R}} .
\end{aligned}
$$

These can be further simplified by noting that both $G_{a b}^{\mathrm{RR}}(n)$ and $G_{a b}^{\mathrm{II}}(n)$ are proportional to $\delta_{a b}$, for all Langevin times. We write therefore

$$
\begin{aligned}
G_{a b}^{\mathrm{RR}}(n) & =\delta_{a b} G^{\mathrm{RR}}(n), \\
G_{a b}^{\mathrm{II}}(n) & =\delta_{a b} G^{\mathrm{II}}(n), \\
G_{a b}^{\mathrm{RI}}(n) & =\delta_{a b} G^{\mathrm{RI}}(n)+\varepsilon_{a b} \bar{G}^{\mathrm{RI}}(n),
\end{aligned}
$$

such that the drift terms reduce to

$$
\begin{aligned}
& K_{a, p}^{\mathrm{R}}=-\mathcal{A}_{p} \phi_{a, p}^{\mathrm{R}}+i B_{p} \varepsilon_{a b} \phi_{b, p}^{\mathrm{I}}+\mathcal{C} \phi_{a, p}^{\mathrm{I}}, \\
& K_{a, p}^{\mathrm{I}}=-\mathcal{A}_{p} \phi_{a, p}^{\mathrm{I}}-i B_{p} \varepsilon_{a b} \phi_{b, p}^{\mathrm{R}}-\mathcal{C} \phi_{a, p}^{\mathrm{R}},
\end{aligned}
$$

with

$$
\mathcal{A}_{p}=A_{p}+4 \lambda\left(G^{\mathrm{RR}}-G^{\mathrm{II}}\right), \quad \mathcal{C}=8 \lambda G^{\mathrm{RI}} .
$$

Since $\mathcal{A}_{p}(n)$ and $\mathcal{C}(n)$ depend explicitly on the Langevin time, the time-dependent mean field Langevin equations cannot be solved analytically. ${ }^{6}$

We therefore look for fixed points. After substituting Eqs. (5.10, 5.11) in the fixed point equations (5.4) and performing some algebra, we find that at the fixed point $\mathcal{C}=0$ and that the two-point functions can be decomposed as

$$
G_{a b, p}^{\mathrm{RR}}=\delta_{a b} G_{p}^{\mathrm{RR}}, \quad G_{a b, p}^{\mathrm{II}}=\delta_{a b} G_{p}^{\mathrm{II}}, \quad G_{a b, p}^{\mathrm{RI}}=i \varepsilon_{a b} G_{p}^{\mathrm{RI}} .
$$

\footnotetext{
${ }^{6}$ In fact, the problem is now very similar to that of nonequilibrium field dynamics using a self-consistent mean field approximation in the equal-time formalism [44].
} 
This is in agreement with the symmetry $2.17,2.20)$. The three fixed point equations (5.4) then become

$$
\begin{array}{r}
\mathcal{A}_{p} G_{p}^{\mathrm{RR}}+B_{p} G_{p}^{\mathrm{RI}}-\frac{\epsilon}{2}\left(\mathcal{A}_{p}^{2} G_{p}^{\mathrm{RR}}+B_{p}^{2} G_{p}^{\mathrm{II}}+2 \mathcal{A}_{p} B_{p} G_{p}^{\mathrm{RI}}\right)=1, \\
\mathcal{A}_{p} G_{p}^{\mathrm{II}}-B_{p} G_{p}^{\mathrm{RI}}-\frac{\epsilon}{2}\left(\mathcal{A}_{p}^{2} G_{p}^{\mathrm{II}}+B_{p}^{2} G_{p}^{\mathrm{RR}}-2 \mathcal{A}_{p} B_{p} G_{p}^{\mathrm{RI}}\right)=0, \\
\mathcal{A}_{p} G_{p}^{\mathrm{RI}}-\frac{1}{2} B_{p}\left[G^{\mathrm{RR}}-G_{p}^{\mathrm{II}}\right]-\frac{\epsilon}{2}\left(\left[\mathcal{A}_{p}^{2}-B_{p}^{2}\right] G_{p}^{\mathrm{RI}}-\mathcal{A}_{p} B_{p}\left[G_{p}^{\mathrm{RR}}-G_{p}^{\mathrm{II}}\right]\right)=0 .
\end{array}
$$

The solution is

$$
\begin{aligned}
G_{p}^{\mathrm{RR}}+G_{p}^{\mathrm{II}} & =\frac{1}{\mathcal{A}_{p}-\frac{1}{2} \epsilon\left(\mathcal{A}_{p}^{2}+B_{p}^{2}\right)}, \\
G_{p}^{\mathrm{RR}}-G_{p}^{\mathrm{II}} & =\frac{1}{\mathcal{A}_{p}^{2}+B_{p}^{2}} \frac{\mathcal{A}_{p}\left(1-\frac{1}{2} \epsilon \mathcal{A}_{p}\right)+\frac{1}{2} \epsilon B_{p}^{2}}{\left(1-\frac{1}{2} \epsilon \mathcal{A}_{p}\right)^{2}+\frac{1}{4} \epsilon^{2} B_{p}^{2}} \\
G_{p}^{\mathrm{RI}} & =\frac{1}{2} \frac{B_{p}}{\mathcal{A}_{p}^{2}+B_{p}^{2}} \frac{1-\epsilon \mathcal{A}_{p}}{\left(1-\frac{1}{2} \epsilon \mathcal{A}_{p}\right)^{2}+\frac{1}{4} \epsilon^{2} B_{p}^{2}}
\end{aligned}
$$

For vanishing Langevin stepsize this simplifies to

$$
G_{p}^{\mathrm{RR}}+G_{p}^{\mathrm{II}}=\frac{1}{\mathcal{A}_{p}}, \quad G_{p}^{\mathrm{RR}}-G_{p}^{\mathrm{II}}=\frac{\mathcal{A}_{p}}{\mathcal{A}_{p}^{2}+B_{p}^{2}}, \quad G_{p}^{\mathrm{RI}}=\frac{1}{2} \frac{B_{p}}{\mathcal{A}_{p}^{2}+B_{p}^{2}},
$$

while in the phase quenched theory $\left(B_{p}=0\right)$ where real Langevin dynamics is applicable, the solution reduces to

$$
G_{p}^{\mathrm{RR}}=\frac{1}{\mathcal{A}_{p}} \frac{1}{1-\frac{1}{2} \epsilon \mathcal{A}_{p}}, \quad G_{p}^{\mathrm{II}}=G_{p}^{\mathrm{RI}}=0 .
$$

We find finite stepsize corrections linear in $\epsilon$, as expected [41]. Furthermore, we note that for large stepsize the denominator in the first line of Eq. (5.15) can go negative. However, this occurs precisely when the stability criterium (3.29) is violated (after the replacement $\left.A_{p} \rightarrow \mathcal{A}_{p}\right)$ and is therefore excluded.

The expressions in Eq. (5.16) agree precisely with the solutions (3.21) obtained by solving the Langevin equations without interactions, after making the mean field replacement $A_{p} \rightarrow \mathcal{A}_{p}$. This replacement corresponds to the standard mean field approximation in which the mass parameter receives a tadpole correction,

$$
m^{2} \rightarrow M^{2}=m^{2}+4 \lambda\left\langle|\phi|^{2}\right\rangle
$$

or, in the notation of this section,

$$
\begin{aligned}
m^{2} \rightarrow M^{2} & =m^{2}+4 \lambda\left(G^{\mathrm{RR}}-G^{\mathrm{II}}\right) \\
& =m^{2}+\frac{4 \lambda}{\Omega} \sum_{p} \frac{\mathcal{A}_{p}}{\mathcal{A}_{p}^{2}+B_{p}^{2}},
\end{aligned}
$$

with

$$
\mathcal{A}_{p}=M^{2}+4 \sum_{i} \sin ^{2} \frac{p_{i}}{2}+2\left(1-\cosh \mu \cos p_{4}\right)
$$



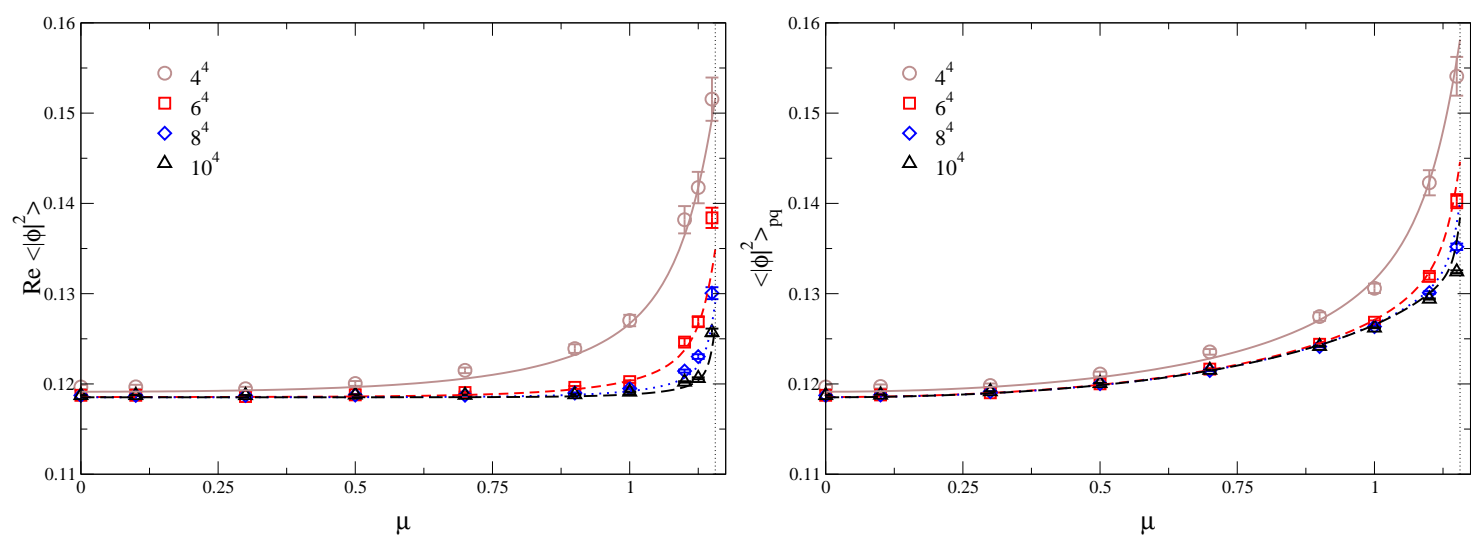

Figure 1: The lines represent the mean field results for $\left\langle|\phi|^{2}\right\rangle$ in the full (left) and phase quenched (right) theories for various lattice sizes, taking $m=\lambda=1$. The vertical dotted line indicates the mean field estimate for the critical chemical potential. The data points are obtained with Langevin simulations 11.
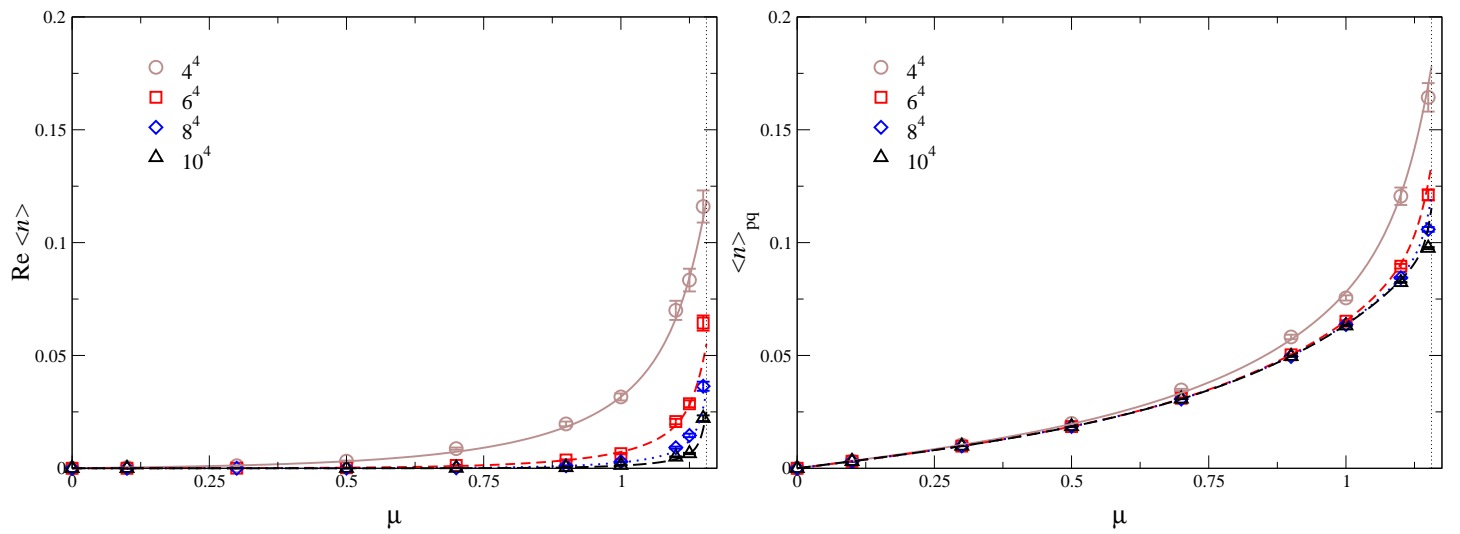

Figure 2: As in Fig. 1 for the density $\langle n\rangle$.

These equations define a self-consistent gap equation for $M^{2}$. Given $m$ and $\lambda$, the gap equation can be solved numerically after specifying the lattice size. For example, taking $m=\lambda=1$, we find $M^{2}=1.47$ and $\left\langle|\phi|^{2}\right\rangle=0.119$ at $\mu=0$ on a lattice of size $10^{4}$. The critical chemical potential then follows from $\mathcal{A}_{0}=0$ (or $M^{2}=4 \sinh ^{2} \frac{\mu}{2}$ ) and is found to be $\mu_{c}=1.15 .^{7}$

We have solved the gap equation in the Silver Blaze region and used the outcome to compute $\left\langle|\phi|^{2}\right\rangle$ and $\langle n\rangle$ in the mean field approximation as a function of chemical potential for different lattice sizes. The results are shown in Figs. 1 and 2 respectively, for $m=$ $\lambda=1$. The vertical dotted lines indicate the mean field estimate of the critical chemical potential. In the full theory (figures on the left) the expected $\mu$ independence emerges in the thermodynamic limit. In the phase quenched theory (figures on the right), observables depend on $\mu$, since there is no Silver Blaze feature, see Appendix A. Also shown in these

\footnotetext{
${ }^{7}$ When $\lambda=0, \mu_{c}$ follows from $A_{0}=0$ (or $m^{2}=4 \sinh ^{2} \frac{\mu}{2}$ ), yielding $\mu_{c}^{0}=0.962$ for $m=1$.
} 

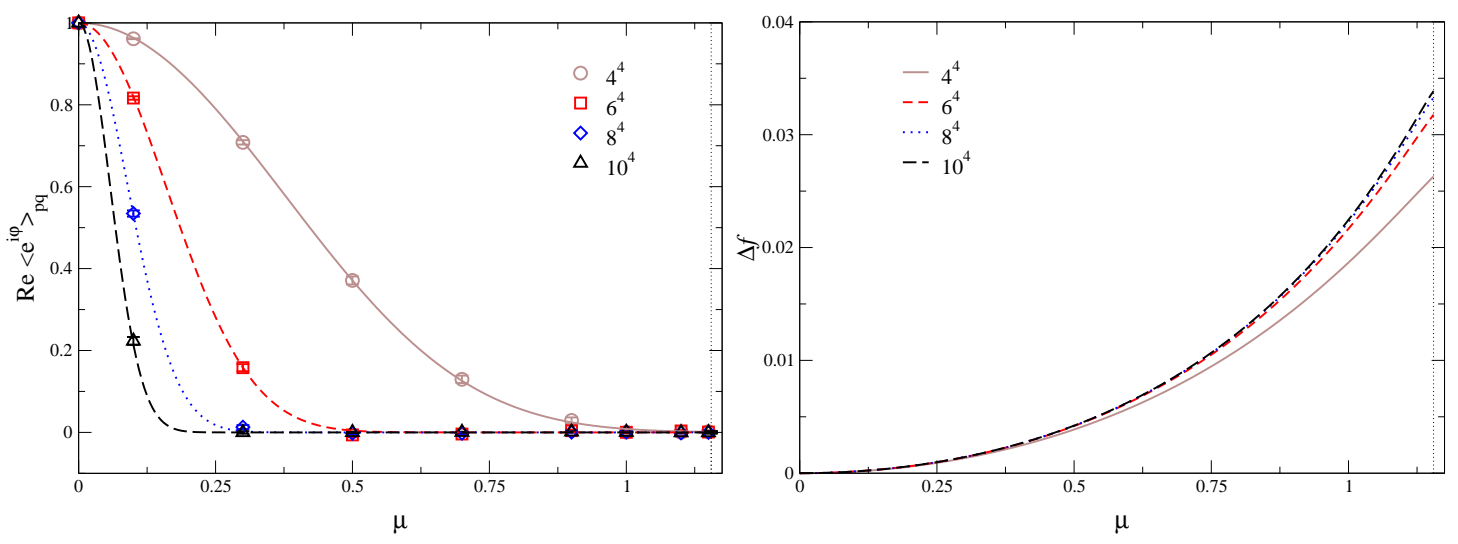

Figure 3: Left: as in Fig. 1 for the average phase factor in the phase quenched theory $\left\langle e^{i \varphi}\right\rangle_{\mathrm{pq}}$. Right: difference $\Delta f$ between the free energy densities of the full and the phase quenched theories in the mean field approximation.

plots are data points obtained from the numerical solution of the Langevin process, with stepsize $\epsilon=5 \times 10^{-5}$ [1]. We observe surprisingly good agreement between the mean field and the nonperturbative results for all values of the chemical potential and all lattice sizes considered, indicating that the mean field approximation captures the most relevant interactions.

In Fig. 3 we show the average phase factor in the phase quenched theory $\left\langle e^{i \varphi}\right\rangle_{\mathrm{pq}}$ (left) and the difference $\Delta f$ between the free energy densities, given in Eq. (3.9), again after the replacement $A_{p} \rightarrow \mathcal{A}_{p}$. As already mentioned, the sign problem is severe in the thermodynamic limit: taking e.g. $\Delta f \sim 0.02$ and a lattice volume $\Omega=10^{4}$, we find that the average phase factor $\left\langle e^{i \varphi}\right\rangle_{\mathrm{pq}}=e^{-\Omega \Delta f}$ is indeed exponentially small.

To conclude this section, we note that it is straightforward to adapt the stationary solution of the extended Fokker-Planck equation, constructed in Sec. 国, to the mean field approximation discussed here. Since in the mean field approximation only two-point functions appear, the mean field probability distribution remains of the form (4.8) with the simple replacement $A_{p} \rightarrow \mathcal{A}_{p}$ (or $m^{2} \rightarrow M^{2}$ ). Existence of the Fokker-Planck distribution in the Silver Blaze region now requires $\mathcal{A}_{p}>0$.

\section{Conclusion}

In order to further understand the applicability of complex Langevin dynamics for theories with a complex action due to finite chemical potential, we have studied the relativistic Bose gas in the Silver Blaze region analytically. Ignoring interactions, we have investigated convergence and stability, and constructed the stationary solution of the extended FokkerPlanck equation. We explained why this real probability distribution is nonlocal in real space. Subsequently, interactions were included on the mean field level and the fixed point of the mean field Langevin equations with finite stepsize was derived. We gave a comparison between the mean field predictions and the nonperturbative numerical data from Ref. [1] in the Silver Blaze region. Surprisingly good agreement was found for all 
values of the chemical potential considered, including finite size effects, indicating that the mean field approximation captures the most important effects of the interactions. We have demonstrated analytically that the sign problem is severe for lattice volumes used in this study. From the combination of results obtained here and in Ref. [1], it can be argued that complex Langevin dynamics in the Silver Blaze region is well understood in this theory.

One obvious next step is to extend the analysis to the high-density phase, which requires the introduction of the mean field $\left\langle\phi_{a, x}^{\mathrm{R}}\right\rangle$ (note that $\left\langle\phi_{a, x}^{\mathrm{I}}\right\rangle=0$ ). Finally, it would be interesting to apply mean field approximations to other theories as well, especially in combination with numerical studies. In particular, this would be useful for QCD with static quarks [36].

\section{Acknowledgments}

I thank Kim Splittorff, Ion-Olimpiu Stamatescu and Simon Hands for their interest and discussion. This work is supported by an STFC Advanced Fellowship.

\section{A. Dispersion relation}

The propagator corresponding to the action (3.2) is

$$
G_{a b, p}=\frac{\delta_{a b} A_{p}+\varepsilon_{a b} B_{p}}{A_{p}^{2}+B_{p}^{2}}
$$

Dispersion relations follow from the poles of the propagator, taking $p_{4}=i E_{\mathbf{p}}$. We find

$$
\cosh E_{\mathbf{p}}(\mu)=\left(1+\frac{1}{2} \hat{\omega}_{\mathbf{p}}^{2}\right) \cosh \mu \pm \sqrt{1+\frac{1}{4} \hat{\omega}_{\mathbf{p}}^{2}} \sinh \mu
$$

where

$$
\hat{\omega}_{\mathbf{p}}^{2}=m^{2}+4 \sum_{i} \sin ^{2} \frac{p_{i}}{2}
$$

This can be written as

$$
\cosh E_{\mathbf{p}}(\mu)=\cosh \left[E_{\mathbf{p}}(0) \pm \mu\right]
$$

such that the (positive energy) solutions are

$$
E_{\mathbf{p}}(\mu)=E_{\mathbf{p}}(0) \pm \mu,
$$

just as in the continuum theory. Lattice discretization effects only appear in the dispersion relation at zero chemical potential, $E_{\mathbf{p}}(0)$. The critical $\mu$ value is $\mu_{c}=E_{\mathbf{0}}(0)=$ $2 \operatorname{asinh}(m / 2)$, so that one mode becomes exactly massless at the transition.

The phase quenched theory corresponds to $B_{p}=0$. In the formal continuum limit, the phase quenched theory is a theory with a real action and mass parameter $m^{2}-\mu^{2}$. The dispersion relation is

$$
\cosh E_{\mathbf{p}}(\mu)=\frac{1}{\cosh \mu}\left(1+\frac{1}{2} \hat{\omega}_{\mathbf{p}}^{2}\right)
$$

corresponding to $E_{\mathbf{p}}^{2}(\mu)=m^{2}-\mu^{2}+\mathbf{p}^{2}$ in the continuum limit, as anticipated.

These results are easily extended to the self-consistent mean field approximation, where the mass parameter receives a tadpole correction and is replaced by $M^{2}=m^{2}+4 \lambda\left\langle|\phi|^{2}\right\rangle$. 


\section{References}

[1] G. Aarts, Phys. Rev. Lett. 102 (2009) 131601 [0810.2089 [hep-lat]].

[2] Z. Fodor and S. D. Katz, Phys. Lett. B 534 (2002) 87 [hep-lat/0104001].

[3] Z. Fodor and S. D. Katz, JHEP 0203 (2002) 014 [hep-lat/0106002].

[4] Z. Fodor, S. D. Katz and K. K. Szabo, Phys. Lett. B 568 (2003) 73 [hep-lat/0208078].

[5] Z. Fodor and S. D. Katz, JHEP 0404 (2004) 050 [hep-lat/0402006].

[6] C. R. Allton et al., Phys. Rev. D 66 (2002) 074507 [hep-lat/0204010].

[7] C. R. Allton, S. Ejiri, S. J. Hands, O. Kaczmarek, F. Karsch, E. Laermann and C. Schmidt, Phys. Rev. D 68 (2003) 014507 [hep-lat/0305007].

[8] C. R. Allton et al., Phys. Rev. D 71 (2005) 054508 [hep-lat/0501030].

[9] R. V. Gavai and S. Gupta, Phys. Rev. D 68 (2003) 034506 [hep-lat/0303013].

[10] P. de Forcrand and O. Philipsen, Nucl. Phys. B 642 (2002) 290 [hep-lat/0205016].

[11] P. de Forcrand and O. Philipsen, Nucl. Phys. B 673 (2003) 170 [hep-lat/0307020].

[12] P. de Forcrand and O. Philipsen, JHEP 0701 (2007) 077 [hep-lat/0607017].

[13] M. D'Elia and M. P. Lombardo, Phys. Rev. D 67 (2003) 014505 [hep-lat/0209146].

[14] Z. Fodor, S. D. Katz and C. Schmidt, JHEP 0703 (2007) 121 [hep-lat/0701022].

[15] S. Ejiri, Phys. Rev. D 78 (2008) 074507 [0804.3227 [hep-lat]].

[16] J. C. Osborn, Phys. Rev. Lett. 93 (2004) 222001 [hep-th/0403131].

[17] G. Akemann, J. C. Osborn, K. Splittorff and J. J. M. Verbaarschot, Nucl. Phys. B 712 (2005) 287 [hep-th/0411030].

[18] J. C. Osborn, K. Splittorff and J. J. M. Verbaarschot, Phys. Rev. Lett. 94 (2005) 202001 [hep-th/0501210].

[19] K. Splittorff and J. J. M. Verbaarschot, Phys. Rev. Lett. 98 (2007) 031601 [hep-lat/0609076].

[20] J. Han and M. A. Stephanov, Phys. Rev. D 78 (2008) 054507 [0805.1939 [hep-lat]].

[21] J. C. R. Bloch and T. Wettig, JHEP 0903 (2009) 100 [0812.0324 [hep-lat]].

[22] S. Chandrasekharan and U. J. Wiese, Phys. Rev. Lett. 83 (1999) 3116 [cond-mat/9902128].

[23] M. G. Endres, Phys. Rev. D 75 (2007) 065012 [hep-lat/0610029].

[24] S. Chandrasekharan, PoS LATTICE2008 (2008) 003 [0810.2419 [hep-lat]].

[25] G. Parisi and Y. s. Wu, Sci. Sin. 24 (1981) 483.

[26] G. Parisi, Phys. Lett. B 131 (1983) 393.

[27] J. R. Klauder and W. P. Petersen, SIAM J. Numer. Anal. 22 (1985) 1153; J. Stat. Phys. 39 (1985) 53.

[28] F. Karsch and H. W. Wyld, Phys. Rev. Lett. 55 (1985) 2242.

[29] J. Ambjorn, M. Flensburg and C. Peterson, Nucl. Phys. B 275 (1986) 375.

[30] P. H. Damgaard and H. Hüffel, Phys. Rept. 152 (1987) 227. 
[31] J. Berges and I. O. Stamatescu, Phys. Rev. Lett. 95 (2005) 202003 [hep-lat/0508030].

[32] J. Berges, S. Borsanyi, D. Sexty and I. O. Stamatescu, Phys. Rev. D 75 (2007) 045007 [hep-lat/0609058].

[33] J. Berges and D. Sexty, Nucl. Phys. B 799 (2008) 306 [0708.0779 [hep-lat]].

[34] C. W. Bernard and V. M. Savage, Phys. Rev. D 64 (2001) 085010 [hep-lat/0106009].

[35] C. Pehlevan and G. Guralnik, Nucl. Phys. B 811, 519 (2009) [0710.3756 [hep-th]].

[36] G. Aarts and I. O. Stamatescu, JHEP 0809 (2008) 018 [0807.1597 [hep-lat]].

[37] T. D. Cohen, Phys. Rev. Lett. 91 (2003) 222001 [hep-ph/0307089].

[38] G. Guralnik and C. Pehlevan, arXiv:0902.1503 [hep-lat].

[39] P. Hasenfratz and F. Karsch, Phys. Lett. B 125 (1983) 308.

[40] J.I. Kapusta, Finite-temperature field theory, Cambridge University Press (1994).

[41] G. G. Batrouni, G. R. Katz, A. S. Kronfeld, G. P. Lepage, B. Svetitsky and K. G. Wilson, Phys. Rev. D 32 (1985) 2736.

[42] J. Ambjorn and S. K. Yang, Phys. Lett. B 165 (1985) 140.

[43] H. Nakazato and Y. Yamanaka, Phys. Rev. D 34 (1986) 492.

[44] G. Aarts, G. F. Bonini and C. Wetterich, Phys. Rev. D 63 (2001) 025012 [hep-ph/0007357]. 